\title{
Análise de Fatores Genéticos e Ambientais que Afetam a Infestação de Fêmeas Bovinas da Raça Caracu por Carrapatos (Boophilus microplus) ${ }^{1}$
}

\author{
Angelina Bossi Fraga², Maurício Mello de Alencar ${ }^{3}$, Leopoldo Andrade de Figueiredo ${ }^{4}$, \\ Alexander George Razook ${ }^{5}$, Joslaine Noely dos Santos Gonçalves Cyrillo 6
}

RESUMO - Este trabalho foi conduzido com o objetivo de avaliar o efeito de fatores de meio sobre a infestação de bovinos Caracu pelo carrapato Boophilus microplus (Canestrini, 1887) e estimar parâmetros genéticos do grau de infestação por esse ectoparasita. Foram realizadas contagens em fêmeas de dois rebanhos, nas quatro estações, por dois anos consecutivos (setembro/ 1998 a julho/2000). Contou-se o número de carrapatos (NC) em um dos lados do animal e atribuiu-se escore visual (EC) de acordo com a quantidade de carrapatos no animal. Foram feitas de uma a oito avaliações, totalizando-se 4.079 e 3.994 observações de NC e EC, respectivamente, em 718 animais. Os dados foram analisados pelo método dos quadrados mínimos com um modelo que incluiu efeitos de rebanho (R), cor do animal (C), R x C, animal dentro de R x C como erro a, ano e estação da avaliação, espessura de pelame e idade do animal como covariável. As estimativas dos componentes de variância foram obtidas pelo método da máxima verossimilhança restrita livre de derivadas, utilizando-se um modelo que incluiu os efeitos fixos de grupo de contemporâneos (fazenda-ano-época), espessura do pelame e idade do animal como covariável e os efeitos aleatórios aditivos diretos e de ambiente permanente. Antes das análises, a variável NC foi transformada para $\log _{10}(\mathrm{n}+1)$ e EC para $(\mathrm{x}+0,5)^{1 / 2}$, em que n é o número de carrapatos contados no animal e x, o escore ( 0 a 4). A incidência de carrapatos foi maior no verão e, quanto maior a espessura do pelame, maior o nível de infestação. As estimativas de herdabilidade e repetibilidade foram, respectivamente, 0,22 e 0,29 para NC e 0,15 e 0,21 para EC; a correlação genética entre NC e EC foi igual a 1,00. Os resultados sugerem que é possível obter progresso genético para resistência a carrapato pela seleção.

Palavras-chave: bovinos, carrapatos, herdabilidade, repetibilidade.

\section{Genetic Analysis of the Infestation of Caracu Female Cattle Breed by Cattle Tick (Boophilus microplus)}

\begin{abstract}
The objective of this study was to evaluate environmental aspects that affect the infestation of the Caracu cattle breed by the cattle tick Boophilus microplus (Canestrini, 1887) and to estimate genetic parameters for the level of this infestation. Countings of the parasite were done on female cattle from two herds, during all four seasons of the year, in two consecutive years (from September 1998 through July 2000). The number of ticks (NC) on one side of the animal was counted, and the infestations were also evaluated by scores (EC), according to the estimated number of parasites on the animal. Each animal had from one to eight observations. A total of 4,079 and 3,994 observations were obtained for NC and EC, respectively. The data were analyzed by the least squares method with a model that included the effects of herd (H), color of the animal (C), $\mathrm{H} \mathrm{x} \mathrm{C}$, animal within $\mathrm{H}$ x $\mathrm{C}$ as error a, year, season, thickness of the hair, and age of animal as a covariable (linear and quadratic effects). The variance and covariance components were estimated by the restricted maximum likelihood derivative free method, using a model that included the effects of contemporary group (herd - year - season), hair thickness and age of the animal as a covariable (linear and quadratic effects), and the random additive direct and permanent environmental effects. Before the analyses, $\mathrm{NC}$ was transformed to $\log _{10}(\mathrm{n}+1)$ and EC was transformed to $(\mathrm{x}+0.5){ }^{1 / 2}$, where $\mathrm{n}$ is the number of ticks counted on the animal and $\mathrm{x}$ is the score for EC ( 0 to 4$)$. The incidence of ticks was higher during the summer, and the ticker the hair the higher the infestation. The heritability and repeatability estimates were equal to 0.22 and 0.29 , and 0.15 and 0.21 for NC and EC, respectively, and the genetic correlation between NC and EC was 1.00. The results indicate that there is enough additive genetic variation on tick numbers to support a selection program to increase resistance.
\end{abstract}

Key Words: Caracu cattle, heritability, repeatability, tick infestation.

\footnotetext{
${ }_{1}^{1}$ Parte da tese de doutorado do primeiro autor, UNESP/Jaboticabal. Bolsista da CAPES.

2 Professora da Universidade Federal de Alagoas, Centro de Ciências Agrárias, Departamento de Zootecnia, Campus Delza Gitaí, BR 104 Norte, km 85, CEP: 57.100-000. E.mail: angelina@ceca.ufal.br

3 Pesquisador da Embrapa Pecuária Sudeste. Bolsista do CNPq. E.mail: mauricio@cppse.embrapa.br

${ }^{4}$ Pesquisador do Instituto de Zootecnia do Estado de São Paulo. E.mail: figueiredo@izsp.br

${ }^{5}$ Pesquisador do Instituto de Zootecnia do Estado de São Paulo. Bolsista do CNPq. E-mail: razook@izsp.br

6 Pós - Graduanda da UNESP/Jaboticabal, Bolsista do CNPq. E.mail: cyrillo@izsp.br
} 


\section{Introdução}

A eficiência da exploração comercial bovina em regiões tropicais depende, em grande parte, do potencial de produção dos animais, bem como da capacidade de adaptação ao ambiente. A ocorrência de infestações parasitárias nos trópicos tem acarretado acentuadas quedas nos índices de produção. A infestação pelo carrapato (Boophilus microplus, Canestrini) assume papel de fundamental importância pois esse parasita se destaca como um dos que mais prejudicam o desempenho dos animais, em conseqüência das ações espoliadora, mecânica e tóxica que exercem sobre eles.

O método de controle de parasitas mais utilizado atualmente é o emprego de produtos químicos, o que acarreta custos elevados e a possível ocorrência de resíduos na carne e no leite. Além desses aspectos, o uso de produtos químicos em grande escala, no combate ao carrapato, pode prejudicar o controle futuro desses parasitas, em decorrência do rápido desenvolvimento de resistência aos princípios ativos utilizados.

Levando-se em conta esses aspectos, justifica-se a utilização de medidas alternativas para complementar os procedimentos de controle tradicionais. Dentro desse contexto, segundo Gavora \& Spencer (1983), a prática da seleção para resistência à infestação parasitária pode ser associada a outras medidas de controle, de modo a resultar no método mais adequado de controle do parasita.

Diferentes níveis de resistência dos bovinos ao Boophilus microplus, tanto entre raças como dentro de raças, foram observados por vários autores, como Wharton et al. (1970), Moraes et al. (1986), Oliveira et al. (1989), Gomes (1992), Andrade (1996) e Veríssimo et al. (1997). Autores como Hewetson (1968), Wharton et al. (1970), Seifert (1971) e Utech (1979), estudando a fração genética aditiva da resistência dos bovinos ao carrapato, encontraram variação genética suficiente para se acreditar na possibilidade de obtenção de considerável progresso genético pela seleção.

No Brasil, alguns trabalhos realizados para estimar a herdabilidade da resistência de bovinos ao carrapato relatam estimativas que variam de 0,00 a 0,30 (Madalena et al.,1985; Guaragna et al., 1988; Gomes, 1992; Veríssimo et al., 1997; Andrade et al., 1998).

Além de fatores genéticos, existem fatores ambientais que podem influenciar no número de pa- rasitas infestantes no hospedeiro, principalmente, pelo fato de que parte do ciclo de vida dos mesmos tem sua ocorrência no meio ambiente. Dentre eles, podem ser citados a estação do ano, o sexo e a idade do animal (Andrade et al.,1998; Cardoso, 2000). De acordo com Penna (1989), a acurácia da avaliação da resistência ao carrapato pode ser reduzida pela ocorrência dos efeitos de ambiente. Portanto, o conhecimento dos fatores de ambiente que influenciam a resistência aos parasitas é importante por ocasião da obtenção de estimativas de parâmetros e valores genéticos, além de auxiliar no manejo sanitário dos rebanhos.

Este trabalho foi conduzido com os objetivos de estudar alguns fatores de ambiente que influenciam os níveis de infestação e estimar parâmetros genéticos e fenotípicos para o grau de infestação de bovinos da raça Caracu por carrapatos (Boophilus microplus), visando ao aumento da resistência a esse ectoparasita.

\section{Materiais e Métodos}

Este trabalho foi realizado com dados de infestação natural por Boophilus microplus em fêmeas da raça Caracu, provenientes de dois rebanhos, pertencentes à Estação Experimental de Zootecnia de Sertãozinho do Instituto de Zootecnia do Estado de São Paulo e à Fazenda Caracu do Ipê Paranaíba, MS, no período entre julho de 1998 até julho de 2000.

O conjunto de dados em estudo foi originado da união dos arquivos de registros de cada local, contendo o total de 4.316 observações. O arquivo de dados da Estação Experimental de Zootecnia de Sertãozinho possuía 1.520 registros, pertencentes a 245 animais, filhos de 39 touros e 166 vacas. O arquivo da Fazenda Caracu do Ipê apresentava 2.796 registros, pertencentes a 792 animais, filhos de 58 touros e 292 vacas.

A avaliação da resistência dos bovinos ao Boophilus microplus baseou-se na contagem de carrapatos fêmeas, infestados naturalmente, de tamanho entre 4,0 e 8,0 $\mathrm{mm}$ de comprimento (NC), denominadas teleóginas, em apenas um dos lados do corpo do animal, método preconizado por Villares (1941) e, posteriormente, modificado por Wilkinson (1955) e Wharton \& Utech (1970). Para efetuar as contagens, os animais foram conduzidos ao tronco de contenção para facilitar o manejo, e submetidos a avaliações repetidas em épocas diferentes (mínimo de 1 e máximo de 8 medidas) com intervalos mínimos de 90 dias após a utilização de produtos antiparasitários. 
O número de teleóginas contadas não foi multiplicado por dois para se ter o total de carrapatos por animal.

Além do método acima descrito, foram atribuídos escores (EC) de 0 a 4, quanto ao nível de infestação dos animais pelo parasita: $0=$ ausência de parasitas (teleóginas e, ou, larvas); 1 = infestação baixa; 2 = infestação média; 3 = infestação alta; e 4 = infestação muito alta. Nessas avaliações, levaram-se em consideração a quantidade de teleóginas e a quantidade de larvas presentes no animal.

Em função de as características estudadas, geralmente, não se ajustarem a uma distribuição normal de probabilidades, as mesmas foram transformadas para: $\log 10(n+1)$, no caso de contagem e $(x+0,5) 1 / 2$, para o escore, em que $n$ é o número de carrapatos contados no animal e $\mathrm{x}$, o escore de infestação de carrapatos (0 a 4$)$.

Os animais foram, ainda, classificados quanto à coloração da capa do pelame em três classes distintas: 1 - baio, 2 - laranja, e 3 - vermelho. A espessura da capa do pelame foi medida com uma régua, modificada artesanalmente, introduzida suavemente sob a superfície da capa do pelame até a base de inserção dos pêlos, na superfície da pele, na região da paleta de cada animal. A espessura de capa do pelame, determinada em classes de $0,5 \mathrm{~cm}$, variou de 0,5 a $6,5 \mathrm{~cm}$; entretanto, as classes $\geq 3,5$ foram agrupadas em uma única classe.

Foram consideradas quatro épocas de contagem de carrapatos: primavera, para as avaliações feitas em setembro, outubro e novembro; verão, para as avaliações feitas em fevereiro e março; outono, para as avaliações feitas em abril e maio; e inverno, para as avaliações feitas em julho.

Após as transformações, as características em estudo foram analisadas pelo método dos quadrados mínimos, utilizando-se o procedimento GLM (SAS, 1996), com o objetivo de verificar possíveis fontes de variação sobre elas. O modelo estatístico utilizado incluiu os efeitos de fazenda $(\mathrm{F}=1 \mathrm{e} 2)$, cor do animal $(\mathrm{C}=1,2$ e 3), F x C, animal dentro de F x C (erro a), ano $(\mathrm{a}=1$ e 2$)$ e estação $(\mathrm{m}=1,2,3,4$; primavera, verão, outono e inverno, respectivamente) da avaliação, espessura do pelame (1 a 3,5) e idade do animal como covariável (efeitos linear e quadrático), além do resíduo.

As estimativas dos componentes de (co)variância, para obtenção dos parâmetros genéticos (herdabilidade e correlação genética), repetibilidades e correlações fenotípicas foram obtidas empregando-se o método da máxima verossimilhança restrita (REML) livre de derivadas, utilizando-se o programa computacional MTDFREML (Boldman et al.,1993). Os efeitos fixos foram compostos por grupo de contemporâneos (GC), formado pela combinação fazenda - ano - estação do ano, pela espessura do pelame e pela covariável idade do animal (efeitos linear e quadrático).

O modelo estatístico utilizado para as análises, em notação matricial, foi:

$$
\mathrm{y}=\mathrm{X}_{\mathrm{b}}+\mathrm{Z}_{\mathrm{a}}+\mathrm{Pp}+\mathrm{e}
$$

em que: $\mathrm{y}=$ vetor de observações; $\mathrm{X}=$ matriz de incidência dos efeitos fixos e da covariável idade do animal; $b=$ vetor dos efeitos fixos (GC e espessura do pelame e idade como covariável); $Z$ = matriz de incidência do efeito genético aditivo direto do animal; $\mathrm{a}=$ vetor dos efeitos genéticos aditivos diretos; $\mathrm{P}=$ matriz de incidência dos efeitos de ambiente permanente e efeitos genéticos não aditivos; $\mathrm{p}=$ vetor de efeitos de ambiente permanente do animal (efeitos não genéticos aditivos, decorrente do próprio animal); e e $=$ vetor de efeitos residuais, aleatórios, associados às observações.

As pressuposições adotadas para o modelo acima descrito foram: $\mathrm{E}(\mathrm{y})=\mathrm{Xb} ; \mathrm{E}(\mathrm{a})=0 ; \mathrm{E}(\mathrm{p})=0 ; \mathrm{E}(\mathrm{e})=0$; $\operatorname{var}(\mathrm{a})=\mathrm{A} \sigma_{\mathrm{a}}^{2} ; \operatorname{var}(\mathrm{p})=\mathrm{I}_{\mathrm{K}} \sigma_{\mathrm{p}}^{2} ; \operatorname{var}(\mathrm{e})=\mathrm{I}_{\mathrm{N}} \sigma^{2}$, sendo que A é o numerador da matriz de parentesco; $\sigma^{2}{ }_{a}$, o componente de variância genética aditiva direta; $\sigma^{2}$, o componente de variância de ambiente permanente; $\sigma^{2}$, o componente de variância residual; $\mathrm{N}$, o número de observações; I, uma matriz identidade de ordem $\mathrm{N}$; e $\mathrm{k}$, o número de animais.

Foram utilizadas 4.079 e 3.994 observações para as análises de NC e EC, respectivamente. Para cada característica foi realizada uma análise unicaráter, usando o modelo animal descrito anteriormente. Foram feitas também análises entre as duas características simultaneamente, para estimar as correlações de NC com EC. A matriz de parentesco continha 2.717 animais.

\section{Resultados e Discussão}

As médias das observações transformadas e em escala normal, os valores mínimos e máximos, os desvios-padrão e os coeficientes de variação das características estudadas podem ser visualizados na Tabela 1. Em virtude das diferenças entre as várias regiões e épocas das avaliações, de efeitos de sexo, raça e idade dos animais e de possíveis diferenças nos controles sanitários, observa-se que o número de 
Tabela 1 - Estatísticas descritivas do número de carrapatos infestantes no animal (NC) e do escore da infestação por carrapatos (EC)

Table 1 - Discriptive statistics of the number of ticks (NC) and of tick score (EC)

\begin{tabular}{|c|c|c|}
\hline $\begin{array}{l}\text { Característica } \\
\text { Trait }\end{array}$ & $\begin{array}{l}\mathrm{NC}^{1} \\
\mathrm{NC}^{1}\end{array}$ & $\begin{array}{l}\mathrm{EC}^{2} \\
E C^{2}\end{array}$ \\
\hline Média ${ }^{3}$ & 1,3333 & 1,2281 \\
\hline$M e a n^{3}$ & $(48,7)$ & $(1,16)$ \\
\hline Mínimo $^{3}$ & 0,0000 & 0,7071 \\
\hline Minimum $^{3}$ & $(0)$ & $(0)$ \\
\hline Máximo $^{3}$ & 3,0800 & 2,1213 \\
\hline Maximum $^{3}$ & (1204) & (4) \\
\hline Desvio-padrão ${ }^{3}$ & 0,6372 & 0,3901 \\
\hline Standard deviation ${ }^{3}$ & $(71,7)$ & $(0,99)$ \\
\hline Assimetria & $-0,4766$ & 0,1215 \\
\hline Assimetry & & \\
\hline Curtose & $-0,3803$ & $-0,7725$ \\
\hline Kurtosis & & \\
\hline $\begin{array}{l}\text { Coeficiente de variação, } \% \\
\text { Coefficient of variation, } \%\end{array}$ & 47,79 & 31,77 \\
\hline
\end{tabular}

carrapatos infestantes nos animais deste trabalho é maior do que aqueles relatados no Brasil por Oliveira et al. (1989), nas raças Nelore e Canchim, Gomes (1992), na raça Gir, Andrade (1996), na raça Gir, e Cardoso (2000), em animais cruzados Zebu x Europeu e Nelore x Angus. Entretanto, está próximo aos valores relatados por Lemos et al. (1985), em novilhas Holandês Vermelho e Branco - Guzerá com contribuição da raça européia maior ou igual a 50\%, Oliveira \& Alencar (1990), em animais de várias composições genéticas de Holandês Vermelho e Branco - Guzerá e Teodoro et al. (1994), em fêmeas filhas de touros das raças Holandesa, Jersey e Pardo - Suiça e vacas de vários composições genéticas de Holandês e Gir, também no Brasil.

As análises de variância (Tabela 2) mostraram efeitos significativos $(\mathrm{P}<0,01)$ apenas da estação do ano e da idade do animal (efeito quadrático) sobre $\mathrm{NC}$ e EC e da espessura do pelame sobre NC. Os outros efeitos incluídos no modelo (fazenda, cor, interação fazenda x cor e ano da avaliação) não influenciaram as características estudadas.

Com relação à cor dos animais, os resultados não significativos encontrados neste trabalho discordam daqueles observados por outros autores. Oliveira \& Alencar (1987), em bovinos da raça Canchim, verificaram que os animais mais claros (brancos) eram mais resistentes ao carrapato do que os mais escuros (amarelos e vermelhos). Os resultados de Veríssimo et al. (1997), em bovinos mestiços leiteiros, sugerem relação entre maior número de carrapatos e cor de pelame escura. Em um rebanho de bovinos da raça Gir, Andrade et al. (1998) verificaram que animais de colorações mais claras como branco, chita e chita claro apresentaram as menores médias de infestação. Segundo esses autores, a maior suscetibilidade ao carrapato dos animais de colorações mais escuras, provavelmente, pode ser provocada pela queda do nível de resistência dos animais mais escuros, em virtude do maior estresse calórico a que estão sujeitos. Neste trabalho, entretanto, não foram encontradas diferenças

Tabela 2 - Resumo das análises de variância do número de carrapatos contados no animal (NC) e do escore de infestação por carrapatos (EC)

Table 2 - Analyses of variance of the number of ticks counted on the animal (NC) and the score of infestation by ticks (EC)

\begin{tabular}{lccc}
\hline $\begin{array}{l}\text { Fonte de variação } \\
\text { Source of variation }\end{array}$ & $\begin{array}{c}\text { Graus de liberdade } \\
\text { Degrees of freedon }\end{array}$ & \multicolumn{1}{c}{$\begin{array}{c}\text { Quadrados médios } \\
\text { Mean squares }\end{array}$} \\
\cline { 2 - 4 } Fazenda (F) (Farm [F]) & 1 & 0,6090 & 0,0085 \\
Cor (C) (Color [C]) & 2 & 0,1144 & 0,1735 \\
Fx C & 2 & 0,4405 & 0,2285 \\
Animal:Fx C & 714 & 0,7301 & 0,2498 \\
Ano (Year) & 1 & 0,6727 & 0,3144 \\
Estação (Season) & 3 & $9,6885^{* *}$ & $4,2993^{* *}$ \\
Esp.do pelame (Hair tickness) & 5 & $2,0928^{* *}$ & 0,1984 \\
Idade (Age) & & & 0,0228 \\
Linear & 1 & 0,4092 & $2,2989^{* *}$ \\
Quadrat. & 1 & $9,6272^{* *}$ & 0,2772 \\
Resíduo (Error) & 3348 & 0,1183 & 36 \\
R $^{2}(\%)$ & 3263 & 44 & 36 \\
\hline
\end{tabular}

${ }^{* *} \mathrm{P}<0,01$ 
no grau de infestação pelo carrapato, em relação à cor dos animais, que variou de baio a vermelho.

Quanto ao efeito da estação do ano, os resultados indicaram a ocorrência de pico de infestação por carrapatos (NC) no verão, com queda pronunciada dessa infestação durante a primavera (Tabela 3). Já para EC, os maiores escores ocorreram para as medidas realizadas no verão e os menores, para as medições feitas no outono, inverno e primavera, não havendo diferenças entre essas últimas (Tabela 3).

$\mathrm{Na}$ literatura, são encontrados resultados bem divergentes quanto à época do ano de maior infestação por ectoparasitas em geral. Autores como Brum et al. (1987) e Andrade et al. (1998) foram unânimes em apontar o outono como a estação que apresenta as maiores médias de infestação por carrapatos. Oliveira et al. (1989) verificaram efeito sazonal significativo, sendo o outono e o inverno as estações de maior infestação. Por outro lado, Utech et al. (1978) observaram que a média de carrapatos no inverno foi significativamente superior em relação à média obtida no verão. Essas diferenças quanto à época do ano de maior infestação por carrapatos, encontradas em vários trabalhos, podem ser atribuídas a um conjunto de fatores específicos de cada região e estação, dentre os quais destacam-se o genótipo do animal, o estado nutricional dos animais, o manejo, as pastagens e as possíveis interações entre eles. Por outro lado, Doube \& Wharton (1980) afirmaram ser possível a existência de um ciclo sazonal na expressão da resistência ao carrapato em bovinos, decorrente da flutuação da capacidade imunitária do animal contra

Tabela 3 - Estimativas das médias ( \pm erro-padrão) do número de carrapatos contados no animal (NC) e escore da infestação por carrapatos (EC), de acordo com a estação do ano

Table 3 - Least square means ( \pm standard error) of the number of ticks (NC) and tick score (EC), according to the season of the year

\begin{tabular}{lcc}
\hline & \multicolumn{2}{c}{ Médias } \\
\cline { 2 - 3 } Estação & $\mathrm{N} C^{1}$ & $\mathrm{EC}^{2}$ \\
Season & $N C^{1}$ & $E C^{2}$ \\
\hline Primavera (Spring) & $1,14 \pm 0,05$ & $1,20 \pm 0,04$ \\
Verão (Summer) & $1,53 \pm 0,03$ & $1,35 \pm 0,02$ \\
Outono (Fall) & $1,46 \pm 0,04$ & $1,20 \pm 0,02$ \\
Inverno (Winter) & $1,43 \pm 0,06$ & $1,21 \pm 0,03$ \\
\hline
\end{tabular}

${ }^{1} \log _{10}(n+1) ;{ }^{2}(x+0,5)^{1 / 2}$

R. Bras. Zootec., v.32, n.6, p.1578-1586, 2003 (Supl. 1) o parasita, a qual se manifesta com queda da resistência no outono e no início do inverno, independentemente da raça e do estado nutricional do animal.

Dentro das condições em que foram realizadas as observações deste trabalho, acredita-se que o pico de infestação por carrapatos no verão pode ter ocorrido em razão de os parâmetros umidade relativa do ar, temperatura e precipitação pluvial serem favoráveis à fase de vida livre do parasita. Outro aspecto que também pode ter contribuído para esse fato é a existência de maior porcentagem de vacas paridas, ou prestes a parir, no verão. Esse estado fisiológico pode acarretar maior suscetibilidade dos animais aos carrapatos. Utech et al. (1978) observaram que as vacas gestantes foram menos resistentes que as não gestantes. Esses autores afirmam, ainda, que o estresse da gestação e as mudanças hormonais podem causar queda da resistência ao carrapato.

Quanto ao efeito da espessura do pelame sobre $\mathrm{NC}$, houve tendência de aumento na contagem de carrapatos com o aumento da espessura do pelame (Tabela 4). De acordo com Bonsma \& Pretorius (1943), animais mais adaptados são aqueles cujos aspectos dos pêlos são curtos, lisos e assentados, enquanto os menos adaptados apresentam pêlos longos, finos e lanosos. Acredita-se, portanto, que animais com menores espessuras de capa de pelame são aqueles mais adaptados e, conseqüentemente, serão aqueles com as menores infestações. Maiores espessuras do pelame podem estabelecer microclima adequado ao parasita, contribuindo para maior possibilidade de sua permanência e sobrevivência no hospedeiro. Além disso, dificulta a prática da auto limpeza realizada pelo animal, sendo este comportamento um dos elementos mais importantes da expressão de resistência. Outro ponto relevante nessa questão é que animais com capa do pelame mais apropriada para regiões tropicais (pêlos curtos, grossos e assentados) e, portanto, capa do pelame com menor espessura apresentam as menores taxas de estresse calórico e, conseqüentemente, maiores níveis de resistência a ectoparasitas. Segundo Veríssimo (1991), o estresse, de modo indireto, pode alterar a resistência dos animais. O aumento dos glicocorticóides na corrente sangüínea ocasionado pelo estresse, em virtude do seu efeito antinflamatório e antialérgico, pode reduzir a reação inflamatória no local de fixação da larva, diminuindo, assim, a reação de auto limpeza do hospedeiro. Esse mecanismo contribui de forma significativa para a permanência do carrapato no animal. 
Tabela 4 - Estimativas das médias ( \pm erro-padrão) do número de carrapatos contados no animal (NC) e do escore da infestação por carrapatos (EC), de acordo com a espessura do pelame

Table 4 - Least square means ( \pm standard error) of the number of ticks (NC) and tick score (EC), according to the hair tickness

\begin{tabular}{lcc}
\hline & \multicolumn{2}{c}{ Médias } \\
\cline { 2 - 3 } Espessura do pelame & $\mathrm{NC}^{1}$ & $\mathrm{EC}^{2}$ \\
Hair tickness & $N C^{1}$ & $E C^{2}$ \\
\hline $1,0(\leq 1,2 \mathrm{~cm})$ & $1,28 \pm 0,03$ & $1,25 \pm 0,02$ \\
$1,5(>1,2 \leq 1,7 \mathrm{~cm})$ & $1,28 \pm 0,02$ & $1,22 \pm 0,01$ \\
$2,0(>1,7 \leq 2,2 \mathrm{~cm})$ & $1,37 \pm 0,02$ & $1,25 \pm 0,01$ \\
$2,5(>2,2 \leq 2,7 \mathrm{~cm})$ & $1,44 \pm 0,03$ & $1,27 \pm 0,02$ \\
$3,0(>2,7 \leq 3,2 \mathrm{~cm})$ & $1,44 \pm 0,04$ & $1,24 \pm 0,02$ \\
$3,5(>3,2 \mathrm{~cm})$ & $1,52 \pm 0,06$ & $1,21 \pm 0,04$ \\
\hline
\end{tabular}

${ }^{1} \log _{10}(\mathrm{n}+1) ;{ }^{2}(\mathrm{x}+0,5)^{1 / 2}$.

As equações de regressão de $\mathrm{NC}$ e EC sobre a idade do animal, que variou de 207 a 6.038 dias, são apresentadas na Tabela 5. O número de carrapatos é máximo em animais de 1.893 dias (5,18 anos) e o escore para carrapatos, máximo para animais de 884 dias (2,42 anos). Esses resultados discordam daqueles observados por Gomes (1992) e Andrade et al. (1998), que verificaram aumento linear no número de carrapatos, com o aumento da idade dos animais.

Na Tabela 6, são apresentadas as estimativas dos componentes de variância, de herdabilidade e de repetibilidade, obtidas pelas análises considerando uma característica de cada vez. A estimativa de herdabilidade obtida para a contagem de carrapatos (NC) indica que a característica apresenta razoável variação genética aditiva, sugerindo a possibilidade de se obter progresso genético pela seleção. Essa estimativa $(0,22)$ é concordante com os valores estimados no Brasil por Madalena et al. (1985), Guaragna et al. (1988) e Andrade et al. (1998) e está acima dos obtidos, também no Brasil, por Gomes (1992) e Veríssimo et al. (1997). Valores mais elevados foram obtidos no exterior por Hewetson (1968), Wharton et al. (1970), Utech (1979) e Mackinnon et al. (1991). Cardoso (2000), no Brasil, obteve estimativas de herdabilidade iguais a 0,00 e 0,47 , para a contagem de carrapatos em animais cruzados Zebu x Europeu, de dois rebanhos. A grande variação dessas estimativas de herdabilidade para resistência à infestação por carrapatos, além de outros fatores, pode ser explicada pela variação de grupo genético do animal, época do ano em que foi realizada a avaliação e ocorrência de contato prévio do animal com o parasita.

A estimativa de herdabilidade para o escore de carrapatos (EC) obtida neste estudo $(0,15)$ é inferior àquela obtida para a contagem de carrapatos $(\mathrm{NC}$; 0,22 ), sugerindo maiores dificuldades de se obter progresso genético por esse método de avaliação da resistência dos animais ao parasita. Cardoso (2000) obteve estimativas de herdabilidade do escore de infestação iguais a 0,00 e 0,21 , para dois rebanhos de animais cruzados.

O componente de variância do efeito de ambiente permanente, que também inclui efeitos genéticos não aditivos, apresentou valores baixos (Tabela 6), porém importantes, para a contagem (NC) e o escore (EC) de carrapatos, pois, somados aos efeitos aditivos, resultaram em valores de repetibilidade de 0,29 e 0,21 , respectivamente, sugerindo que existe algum efeito hereditário e, ou, de ambiente permanente, com respeito a diferenças entre animais para essas características. Entretanto, para maior segurança, deve-se fazer mais de uma observação de contagem ou de escore de carrapatos na avaliação dos animais. No caso de NC, a primeira contagem seria cerca de $29 \%$ acurada para predizer a segunda contagem, enquanto que a média das duas primeiras contagens seria $45 \%$ acurada para predizer a terceira, o que representa aumento de $55 \%$ na acurácia tomando-se duas medidas em vez de apenas uma. O valor de repetibilidade obtido neste estudo para $\mathrm{NC}$ é mais baixo do que o valor de 0,45 estimado por Mackinnon et al. (1991).

$\mathrm{Na}$ Tabela 6 são apresentadas também as estimativas de herdabilidade, de repetibilidade e das correlações genética, de ambiente permanente (não genética aditiva), residual e fenotípica para as características estudadas, obtidas pelas análises de duas carac-

Tabela 5 - Equações de regressão do número de carrapatos contados no animal (NC) e do escore da infestação por carrapatos (EC) sobre a idade do animal

Table 5 - Regression equations of the number of ticks (NC) and tick score (EC) on animal age

\begin{tabular}{lc}
$\begin{array}{l}\text { Característica } \\
\text { Trait }\end{array}$ & $\begin{array}{c}\text { Equação de regressão } \\
\text { Regression equation }\end{array}$ \\
\hline $\mathrm{NC}$ & $0,5050+0,000473 \times$ Idade $-0,000000125 \times$ idade $^{2}$ \\
$N C$ & $0.5050+0.000473 \times$ age $-0.000000125 \times$ age $^{2}$ \\
$\mathrm{EC}$ & $1,3444+0,000113 \times$ Idade $-0,000000064 \times$ idade $^{2}$ \\
$E C$ & $1.3444+0.000113 \times$ age $-0.000000064 \times$ age $^{2}$ \\
\hline
\end{tabular}


Tabela 6 - Componentes de variância, herdabilidade, repetibilidade e correlações genética e fenotípica do número de carrapatos (NC) e escore da infestação por carrapatos (EC), obtidos pelas análises de uma característica de cada vez e das duas características simultaneamente

Table 6 - Variance components, heritability, repeatability and genetic and phenotypic correlations of the number of ticks (NC) and tick score (EC), obtained by one-trait and two-trait analyses

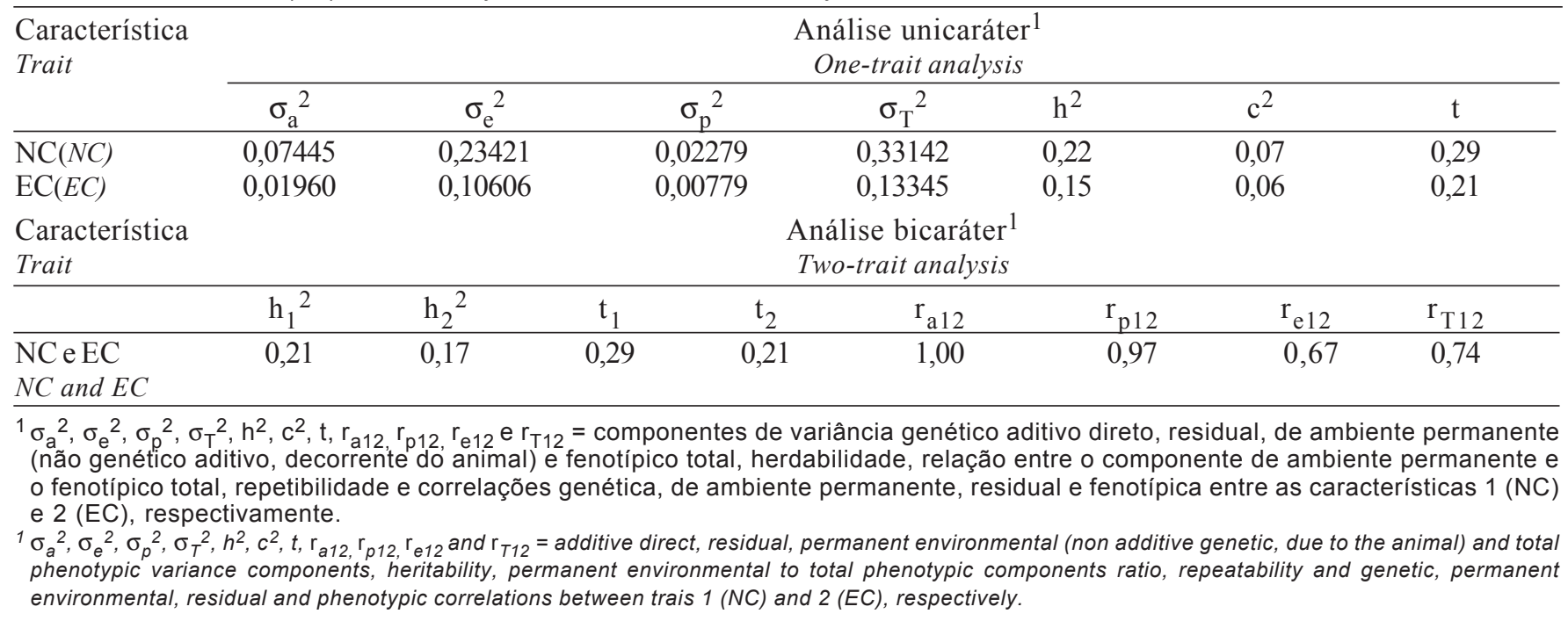

terísticas simultaneamente. Observa-se que as estimativas de herdabilidade e de repetibilidade são iguais ou muito semelhantes àquelas obtidas pelas análises de uma característica de cada vez.

A estimativa de correlação genética de NC com EC é perfeita, sugerindo que trata-se da mesma característica, porém, avaliada de formas distintas e que a seleção para uma delas, deve resultar em resposta correlacionada na outra. Por outro lado, Cardoso (2000) observou correlação genética igual a -0,35 entre o número de carrapatos do lado esquerdo do animal e o escore de infestação (com variação de escore $1=$ infestação elevada a escore 5 = infestação nula), concluindo que as avaliações por escore são ineficientes na estimativa da resistência ao carrapato.

Apesar de a herdabilidade para EC ser menor do que a herdabilidade para $\mathrm{NC}$, provavelmente seja vantajosa a utilização do escore (EC; 0 a 4) em lugar de NC, em virtude do tempo que se gasta contando os parasitas no animal. Considerando-se as estimativas de herdabilidade e de correlação genética obtidas neste estudo e, ainda, a mesma intensidade de seleção para as duas características, a seleção para EC é cerca de $90 \%$ tão eficiente quanto a seleção para $\mathrm{NC}$, para promover mudanças em NC.

A correlação de ambiente permanente, ou correlação não genética aditiva decorrente do animal, foi próxima à unidade (Tabela 6). Isto indica que os fatores não genéticos aditivos próprios do animal, ou seja, aqueles ligados ao ambiente não temporário e às combinações gênicas dentre e entre locos, que influenciam uma característica também influenciam a outra.

A relação entre as correlações fenotípica, genética aditiva, de ambiente permanente (não genética aditiva decorrente do animal) e de ambiente (residual ou de ambiente não permanente ou temporário) pode ser expressa pela seguinte função:

$\mathrm{rP}_{12}=\mathrm{h}_{1} \cdot \mathrm{ra}_{12} \cdot \mathrm{h}_{2}+\mathrm{c}_{1} \cdot \mathrm{rpe}_{12} \cdot \mathrm{c}_{2}+\mathrm{e}_{1} \cdot \mathrm{re}_{12} \cdot \mathrm{e}_{2}$ em que $h_{i}=$ raiz quadrada de $h_{i}^{2}$ (herdabilidade da característica $\mathrm{i}$ ); $\mathrm{c}_{\mathrm{i}}=$ raiz quadrada de $\mathrm{c}_{\mathrm{i}}^{2}$ (relação entre o componente de variância de ambiente permanente e o fenotípico total); ei $=$ raiz quadrada de $\mathrm{e}_{\mathrm{i}}{ }^{2}$ (relação entre o componente de variância residual ou de ambiente não permanente ou temporário e o fenotípico total); e $\mathrm{rP}_{12}, \mathrm{ra}_{12}, \mathrm{rpe}_{12}$ e $\mathrm{re}_{12}=$ correlações fenotípica, genética aditiva, de ambiente permanente e residual, respectivamente. Considerando-se essa relação entre as correlações, as contribuições dos componentes genético aditivo, não genético aditivo decorrente do animal (genéticos não aditivos e de ambiente permanente) e de ambiente (residual ou temporário) para a correlação fenotípica entre as características foram iguais a 25,23; 7,83 e 66,94\%, respectivamente. Observa-se que, apesar de a correlação de ambiente permanente entre as características ser próxima da unidade (Tabela 6), o componente de ambiente permanente contribuiu muito pouco para a correlação fenotípica entre as duas características. 
Já o componente de ambiente (residual) contribuiu com a maior parte dessa correlação e a contribuição do componente genético aditivo foi importante, apesar de não ter sido a maior.

\section{Conclusões}

A maior incidência de carrapatos em bovinos Caracu foi verificada durante o verão.

A espessura do pelame do animal é fator importante na infestação de carrapatos, sendo tanto maior a infestação quanto maior for a espessura do pelame.

Existe variação genética aditiva para o grau de infestação por carrapatos suficiente para se obter progresso genético para resistência, pela seleção de animais menos infestados.

Existem efeitos genéticos (aditivos e não aditivos) e, ou, de ambiente permanente, responsáveis por diferenças entre animais para o grau de infestação por carrapatos; entretanto, deve-se fazer mais de uma contagem de carrapatos por ocasião da avaliação dos animais.

O escore de infestação por carrapatos pode substituir a contagem do parasita no corpo do animal com pequena perda de eficiência quanto à resposta à seleção.

\section{Literatura Citada}

ANDRADE, A.B.F. Aspectos genéticos e ambientais da resistência a Boophilus microplus de bovinos da raça Gir, da Estação Experimental da EPAMIG, Uberaba, MG, Brasil. Jaboticabal: Universidade Estadual Paulista, 1996. 79p. Dissertação (Mestrado em Zootecnia) - Universidade Estadual Paulista, 1996.

ANDRADE, A.B.F.; SILVA, R.G.; COSTA, A.J. et al. Genetic and Environmental aspects of the resistance of zebu cattle to the tick Boophilus microplus. In: WORLD CONGRESS ON GENETICS APPLIED TO LIVESTOCK PRODUCTION, 6., 1998, Armidale. Proceedings... Armildade, n.27, p.339-342, 1998.

BOLDMAN, K.G.; KRIESE, L.A.; Van VLECK, L.D. et al. A manual for Use of MTDFREML. A set of Programs to Obtain Estimates of Variances and Covariances. Lincoln: Department of Agriculture, Agricultural Research Service, 1993.

BONSMA, J.C.; PRETORIUS, A.J. Influence of color coat cover on adaptability of cattle. Farming in South Africa, v.18, p.101-120, 1943.

BRUM, J.G.W.; COSTA, P.R.P.; RIBEIRO, P.B. et al. Flutuação Sazonal de Boophilus microplus (Canestrini, 1887) no município de Pelotas, RS. Arquivo Brasileiro de Medicina Veterinária e Zootecnia, v.39, n.6, p.891-896, 1987.

CARDOSO, V. Avaliação de diferentes métodos de determinação da resistência genética ao carrapato Boophilus microplus, em bovinos de corte. Jaboticabal: Universidade Estadual de São Paulo, 2000. 108p. Dissertação (Mestrado em Zootecnia) - Universidade Estadual de São Paulo, 2000.

DOUBE, B.M.; WHARTON, R.H. The effect of locality, breed and previous tick experience on seasonal change in the resistance of cattle to Boophilus microplus (Ixodoidea: Ixodidae). Experientia, v.36, p.1178-9, 1980.

GAVORA, J.S.; SPENCER, L. Breeding for immune responsiveness and diasease resistance. Animal Blood Groups Biochemical. Genetics, v.14, p.159-180. 1983.

GOMES, A. Resistência a infestação natural por larvas, ninfas e adultos de Boophilus microplus em vacas zebuínas da raça Gir, em função de sua idade, da gestação, da lactação e da seleção para produção leiteira, com e sem tratamento carrapaticida, ao longo de 12 estações consecutivas de um triênio. São Paulo: Universidade de São Paulo, 1992. 90p. Tese (Doutorado em Ciência) - Universidade de São Paulo, 1992.

GUARAGNA, G.P.; CARVALHO, J.B.P.; FIGUEREDO, A.L. et al. Efeitos de fatores genéticos e ambientes na infestacão natural de carrapatos (Boophilus microplus, canestrini) em bovinos leiteiros. Boletim Indústria Animal, v.45, n.1, p.19-32, 1988.

HEWETSON, R.W. Resistance of cattle to cattle tick Boophilus microplus. II The Inheritance of resistance to experimental infestations. Australian Journal Agricultural Research, v.19, p.495-505, 1968.

LEMOS, A.M.; TEODORO, R.L.; OLIVEIRA, G.P. et al. Comparative performance of six Holstein-Friesian x Guzerá grades in Brazil. 3. Burdens of Boophilus microplus under field conditions. Animal Production, v.41, n.2, p.187-91, 1985.

MACKINNON, M.J.; MEYER, K.; HETZEL, D.J.S. Genetic variation and covariation for growth, parasite resistance and heat tolerance in tropical cattle. Livestock Production Science, v.27, p.105-122, 1991.

MADALENA, F.E.; TEODORO, R.L.; LEMOS, A.M. et al. Causes of variation of field burdens of cattle ticks (B. microplus). Revista Brasileira de Genética, v.8, n.2, p.361-375, 1985.

MORAES, F.R.; COSTA, A.J.; WOELZ, C.R. et al. Ecologia de Carrapatos XV: Suscetibilidade Natural Comparativa entre taurinos e Zebuínos a Boophilus microplus (Canestrini) (Acari, Ixodidae). Ars Veterinária, v.2, n.1, p.45-52, 1986.

OLIVEIRA, G.P.; ALENCAR, M.M. Resistência de bovinos ao carrapato Boophilus microplus. I Infestação Artificial. Pesquisa Agropecuária Brasileira, v.22, n.4, p.433-438, 1987.

OLIVEIRA, G.P.; ALENCAR, M.M.; FREITAS, A.R. Resistência de bovinos ao carrapato Boophilus microplus. II Infestação Natural. Pesquisa Agropecuária Brasileira, v.24, n.10, p.1267-1271, 1989.

OLIVEIRA, G.P.; ALENCAR, M.M. Resistência de bovinos de seis graus de sangue Holandes-Guzerá ao carrapato (Boophilus microplus) e ao berne (Dermatobia hominis). Arquivo Brasileiro de Medicina Veterinária e Zootecnia, v.42, n.2, p.127-135, 1990.

PENNA, V.M. Boophilus microplus: A resistência genética do hospedeiro como forma de controle. Caderno Técnico Escola Veterinária UFMG, v.4, p.65, 1989.

SEIFERT, G.W. Variations between and within breeds of cattle in resistance to field infestations of the cattle tick (Boophilus microplus). Australian Journal Agricultural Research, v.22, p.159-68, 1971. 
STATISTICAL ANALYSIS SYSTEMS - SAS. Statistical analysis systems user's guide. 4.ed. v.2. 1996.

TEODORO, R.L.; LEMOS, A.M.; MADALENA, F.E. Carga parasitária de Boophilus microplus em vacas mestiças Europeu $x$ Zebu. Revista da Sociedade Brasileira de Zootecnia, v.23, n.2, p.223-228, 1994.

UTECH, K.B.W. Prospects of selection for tick resistance in british breeds of cattle. The future of the british breeds in ticks areas. Queensland Agricultural College. p.30-8, 1979.

UTECH, K.B.W.; SEIFERT, G.W.; WHARTON, R.H. Breeding Australian Illawarra Shorthorn cattle for resistance to Boophilus microplus. 1. Factors affecting resistance. Australian Journal Agricultural Research, v.29, p.411-422, 1978.

VERÍSSIMO, C.J. Resistência e suscetibilidade de bovinos Leiteiros mestiços ao Carrapato Boophilus microplus. Jaboticabal: Universidade Estadual de São Paulo, 1991. 169p. Dissertação (Mestrado em Zootecnia) - Universidade Estadual de São Paulo, 1991.

VERÍSSIMO, C.J.; SILVA, R.G.; OLIVEIRA, A.A.D. et al. Resistência e suscetibilidade de bovinos leiteiros mestiços ao carrapato Boophilus microplus. Boletim Indústria Animal, v.54, n.2, p.1-10, 1997.
VILLARES, J.B. Climatologia zootécnica. III. Contribuição ao estudo da resistência e susceptibilidade genética dos bovinos ao Boophilus microplus. Boletim Indústria Animal, v.4, n.1, p.60-86, 1941.

WHARTON, R.H.; UTECH, K.B.W.;TURNER, H.G. Resistance to the cattle tick, Boophilus microplus in a Herd of Australian Illawarra Shorthorn cattle: Its Assessment and Heritability. Australian Journal Agricultural Research, v.21, p.163-181, 1970.

WHARTON, R.H.; UTECH, K.B.W. The relation between engorgement and dropping of Boophilus microplus (Canestrini) (Ixodidae) to the assesment of tick numbers on cattle. Journal Australian Entomological Society, v.9, p.171-182, 1970.

WILKINSON, P.R. Observations on infestation of undipped cattle of britsh breeds with the cattle tick, Boophilus microplus (Canestrini). Australian Journal Agricultural Research, v.6, p.655-665, 1955.

Recebido em: 03/10/02

Aceito em: 10/04/03 